\title{
Regional Neural Activity Changes in Parkinson's Disease-Associated Mild Cognitive Impairment and Cognitively Normal Patients
}

\author{
Yilan Xing (1D ${ }^{1,2}$ \\ Shishun $\mathrm{Fu}^{3}$ \\ Meng $\mathrm{Li}^{3}$ \\ Xiaofen $\mathrm{Ma} \mathbb{1}^{3}$ \\ Mengchen $\mathrm{Liu}^{3}$ \\ Xintong Liu $^{2}$ \\ Yan Huang ${ }^{2}$ \\ Guang $X \mathrm{u}^{2}$ \\ Yonggang Jiao ${ }^{2}$ \\ Hong $\mathrm{Wu}^{2}$ \\ Guihua Jiang $\mathbb{D}^{1,3}$ \\ Junzhang Tian ${ }^{1,3}$
}

'The Second School of Clinical Medicine, Southern Medical University, Guangzhou, People's Republic of China; ${ }^{2}$ Department of Neurology of Guangdong Second Provincial General Hospital, Guangzhou, People's Republic of China; ${ }^{3}$ Department of Medical Imaging of Guangdong Second Provincial General Hospital, Guangzhou, People's Republic of China
Correspondence: Junzhang Tian; Guihua Jiang

Department of Medical Imaging of Guangdong Second Provincial General Hospital, No. 466, Xingang Road, Guangzhou, People's Republic of China Tel +8620 89I6870I; +86 I8620I20333 Email tianjunzhang2015@163.com; GH. jiang2002@163.com
Purpose: The aim of this study was to compare regional homogeneity (ReHo) changes in Parkinson's disease mild cognitive impairment (PD-MCI) patients with respect to normal controls (NC) and those with cognitively normal PD (PD-CN). Further, the study investigated the relationship between ReHo changes in PD patients and neuropsychological variation.

Patients and Methods: Thirty PD-MCI, 19 PD-CN, and 21 NC subjects were enrolled. Resting state functional magnetic resonance imaging data of all subjects were collected, and regional brain activity was measured for ReHo. Analysis of covariance for ReHo was determined between the PD-MCI, PD-CN, and NC groups. Spearman rank correlations were assessed using the ReHo maps and data from the neuropsychological tests.

Results: In comparison with NC, PD-CN patients showed significantly higher ReHo values in the right middle frontal gyrus (MFG) and lower ReHo values in the left supramarginal gyrus, bilateral inferior parietal lobule (IPL), and the right postcentral gyrus (PCG). In comparison with PD-CN patients, PD-MCI patients displayed significantly higher ReHo values in the right PCG, left middle occipital gyrus (MOG) and IPL. No significant correlation between ReHo indices and the neuropsychological scales was observed.

Conclusion: Our finding revealed that decreases in ReHo in the default mode network (DMN) may appear before PD-related cognitive impairment. In order to preserve executive attention capacity, ReHo in the right MFG in PD patients lacking cognition impairment increased for compensation. PD-MCI showed increased ReHo in the left MOG, which might have been caused by visual and visual-spatial dysfunction, and increased ReHo in the left IPL, which might reflect network disturbance and induce cognition deficits.

Keywords: Parkinson's disease, mild cognitive impairment, ReHo, fMRI, DMN

\section{Plain Language Summary}

- Regional neural activity changes were observed among Parkinson's disease mild cognitive impairment (PD-MCI) patients, Parkinson's disease cognitively normal patients and normal controls using regional homogeneity (ReHo) analysis.

- Decreased ReHo values in default mode network (DMN) may appear before cognition impairment.

- Increased ReHo values in the left middle occipital gyrus, the left inferior parietal lobule, and the right postcentral gyrus of PD-MCI patients may be contribute to cognitive dysfunction.

- The ReHo changes may provide useful information for the early detection of PD-MCI. 


\section{Introduction}

Parkinson's disease (PD) is a common neurodegenerative disease characterized by motor and non-motor symptoms. Cognitive impairment, anxiety, depression, apathy, psychosis, and sleep disorders are the important non-motor symptoms. Among these, cognitive impairment, especially mild cognitive impairment (MCI), has become a research hotspot. Evidence from longitudinal and cross-sectional research reveals that about $20-53 \%$ of PD patients suffer from MCI. ${ }^{1,2}$ The prevalence of PD-MCI is about 22.8 $46.8 \%$ in Asian countries. ${ }^{2}$ PD-MCI is a transitional stage between cognitively normal PD (PD-CN) and PD dementia (PDD), and most patients with PD-MCI progress to PDD within 3-5 years. Among those with PD-MCI, only a few patients reacquire normal cognition. ${ }^{1}$ Executive dysfunctions comprise the most frequently observed cognitive symptoms, ${ }^{2}$ although memory impairment is also very common. Since PD-MCI may be a precursor to PDD, which impairs quality of life, increases disability and mortality, and intensifies caregiver burden, determining the neural substrates of PD-MCI would be crucial for identifying PD patients with high risk of further cognitive deterioration in order to initiate preventive treatment as soon as possible. Up to now, the neurophysiological mechanisms of PD-MCI have yet to be characterized.

A variety of neuroimaging tools have been applied to investigating the neural mechanisms of PD-MCI. Positron emission tomography, single-photon emission computed tomography, and arterial spin labeling MRI (ASL-MRI) are capable of measuring cerebral blood flow and showing posterior cortical hypoperfusion in PD-nondemented patients. ${ }^{3}$ Voxel-based morphometry and cortical thickness analyses visualize atrophy of temporal, occipital, parietal, and frontal cortices in PD-MCI. ${ }^{4}$ In addition, resting-state fMRI (RS-fMRI) has been more and more widely used in PD-MCI. Functional connectivity (FC) studies reveal hypoconnectivity mainly within the default mode network $(\mathrm{DMN})$, which affects reciprocal interaction with other cerebral networks in cases of PD-MCI. ${ }^{5}$ Independent component analysis (ICA) is another study that has shown hyperconnectivity between $\mathrm{DMN}$ and posterior-cortical region. ${ }^{4}$ Except for resting-state brain $\mathrm{FC}$ and largeworld analyses, regional homogeneity (ReHo) has been the chief procedure employed for detecting local neural synchronization by measuring similarities of intra-regional time series across the whole brain using regional blood oxygenation level-dependent (BOLD) signaling. ReHo showed test-retest reproducibility in nearly all gray matter $^{6}$ and was characterized by being purely datadriven and by its sensitivity, like ICA. ${ }^{7}$

ReHo was first proposed by Zang and colleagues in 2004 in cognitive tasks. At present, ReHo is a valuable tool that has been successfully used to detect local neural synchronization in normal aging subjects and neurodegenerative diseases such as $\mathrm{MCI}$ and $\mathrm{PD} .^{8-10}$ Comparisons between PD-CN patients and normal controls (NC) revealed increased ReHo alterations in the DMN, sensorimotor network, basal ganglia, left angular gyrus, and left precentral gyrus ${ }^{9,11-13}$ and reduced ReHo alterations in the frontal, occipital, primary sensory cortex, visual network and cerebellar regions. ${ }^{11,12,14,15}$ PD sub-types without cognitive impairment, including tremor-dominant (TD) and postural instability gait difficulty-dominant/akinetic-rigiddominant (PIGD) PD patients, showed abnormal ReHo indices in the DMN. ${ }^{11,12,14,15}$ Besides the cross-sectional study, a longitudinal study with a 2-year follow-up on PD patients with normal conditions exhibited decreased ReHo in the DMN over time. ${ }^{17}$ Only a few studies have applied ReHo for detecting local neural synchronization in cases of PD-MCI. ${ }^{11,14}$ For example, comparison between PDMCI patients and NC showed increased ReHo alterations in the limbic lobe and left fusiform gyrus, decreased ReHo alterations in the left lingual gyrus, left thalamus, left cerebellum and right supplementary motor area. ${ }^{11,14} \mathrm{On}$ the whole, previous reports have chiefly focused on comparisons between PD-MCI patients and $\mathrm{NC}$ or PD-CN patients and NC. The comparison of PD-MCI, PD-CN, and $\mathrm{NC}$ groups in one study is helpful for investigating PD-MCI- and PD-CN-related regional brain neural activity accurately to have a deeper understanding of the pathophysiology of PD-MCI. To date, two research using the ReHo method has explored changes in local brain function in PD-MCI compared with PD-CN patients and NC. One research reported increased spontaneous synchrony in the $\mathrm{DMN}^{14}$ while the other one presented opposite result in comparison with PD-MCI and PD-CN patients. ${ }^{11}$ Moreover, both of the two research did not record the levodopa equivalent daily dose (LEDD) of the PD patients. ${ }^{14}$

Therefore, the main goal of this study was to examine local neural synchronizations in PD-MCI and to compare them to those of PD-CN patients and $\mathrm{NC}$ using ReHo analysis, behavioral scales, and such clinical data as LEDD, clinical type, and disease duration. We hypothesized that ReHo alterations in the DMN would differ 
between the PD-MCI group and the PD-CN and NC groups and that ReHo alterations would be correlated with neuropsychiatric parameters.

\section{Patients and Methods}

\section{Participants}

In total, 49 patients were recruited from June 2017 to July 2018 from among inpatients and outpatients of the Department of Neurology, Guangdong Second Provincial General Hospital. Thirty patients were diagnosed as PD$\mathrm{MCI},{ }^{18}$ and 19 patients were diagnosed as PD-CN. All participants were right-handed.

The inclusion criteria for patients were as follows: (1) a clinical diagnosis of idiopathic PD using the UK PD Society Brain Bank diagnostic criteria ${ }^{19}$ and (2) an age range from 40 to 80 years.

The exclusion criteria for patients were as follows: (1) PDD and other types of dementia (Alzheimer's disease, dementia with Lewy body, frontotemporal dementia, and vascular dementia), (2) A revised Hoehn and Yahr (H-Y) score > III, (3) Significant psychiatric, neurological, or systemic comorbidity, (4) Contraindications as assessed using MRI scanning, (5) Oral intake of psychotropic agents and anticholinergic drugs, and (6) Secondary parkinsonism and parkinsonism-plus syndromes.

Twenty-one age, sex matched NC were recruited. The exclusion criteria included (1) the presence of MCI, (2) depression or dysthymic disorder (American Psychiatric Association (1994) Diagnostic and statistical manual of mental disorders, 4th edition, American Psychiatric Association, Washington, DC), (3) significant neurological or systemic disease, and (4) contraindications to MRI scanning. The study was approved by the Ethics Committee of Southern Medical University and the Second School of Clinical Medicine Institutional Review Board, which was carried out in accordance with the Declaration of Helsinki. All subjects provided written informed consent.

\section{Clinical and Neuropsychological Assessment}

The demographics, family history and past history of subjects, disease duration, clinical types, LEDD, presence with hyposmia, values of uric acid, and homocysteine of patients were recorded by neurological specialists.

The Montreal Cognitive Assessment (MoCA) and the Mini-Mental State Examination (MMSE) were administered to evaluate the global cognition of all subjects. Cutoff points for the MMSE were $>17$ for illiteracy, $>20$ for primary education, and $>24$ for junior and higher education.

PD-MCI was diagnosed according to the Movement Disorder Society Task Force, Level 1 diagnostic criteria, which simplified classification by not providing PD-MCI subtypes. PD-MCI defines subjective or objective cognitive impairment as an MoCA $<26$ and an absence of daily living activity (ADL) dysfunctions. ${ }^{20} \mathrm{PD}-\mathrm{CN}$ was defined as an MoCA $\geq 26$ and an absence of ADL dysfunctions.

The Unified Parkinson's Disease Rating Scale motor subscore (UPDRS-III) and Revised H-Y Scale were administered to evaluate the motor status and disease severity of the patients. The Hamilton Depression Rating Scale (HAMD) and the Hamilton Anxiety Rating Scale (HAMA) were administered to evaluate depressive and anxiety symptoms.

\section{MRI Data Acquisition}

An RS-MRI was obtained from each of the participants using a 3.0-T MR imager (Ingenia; Philips, Best, The Netherlands) equipped with a 32-channel head coil at the Department of Medical Imaging in Guangdong Second Provincial General Hospital. The RS-fMRI data were acquired using gradient echo-planar imaging (EPI) set to the following parameters: repetition time (TR)/echo time $(\mathrm{TE})=2000 \mathrm{~ms} / 30 \mathrm{~ms}$; acquisition matrix = $64 \times 64$; field of view $(\mathrm{FOV})=230 \mathrm{~mm} \times 230 \mathrm{~mm}$; flip angle (FA) $=90^{\circ}$; slice thickness $=3.6 \mathrm{~mm}, 0.6 \mathrm{~mm}$ gap; interleaved scanning; and 38 transverse slices covering the whole brain. Two hundred forty volumes were obtained for each participant within $480 \mathrm{~s}$, and each volume was aligned along the anterior-posterior commissure. Additionally, T1-weighted and T2-FLAIR images were obtained in order to detect any brain lesions. The T1weighted images were obtained using a fast field echo (FFE) pulse sequence set according to the following parameters: $\mathrm{TR} / \mathrm{TE}=25 \mathrm{~ms} / 4.1 \mathrm{~ms} ; \mathrm{FA}=30^{\circ}$; acquisition matrix $=256 \times 256 ; \mathrm{FOV}=230 \mathrm{~mm} \times 230 \mathrm{~mm}$; and slice thickness $=1.0 \mathrm{~mm}$. One hundred sixty sagittal slices were obtained. Each participant was instructed to lie still and to avoid falling asleep or having extraneous thoughts during MR scanning.

\section{Data Processing and ReHo Calculations}

All EPI data were processed using a DPARSF 3.0 Advanced Edition (http://rfmri.org/DPARSF). The first 10 volumes were discarded to allow for magnetic field 
stabilization and adaptation of the participants to fMRI noise. The remaining RS-fMRI datasets were corrected for intra-volume acquisition time delay, inter-volume head motion, and co-registration with the anatomical scan. The co-registered anatomical images were segmented into gray matter, white matter, and cerebrospinal fluid. Functional images were then normalized with respect to the Montreal Neurological Institute (MNI) space with an isotropic voxel size of $3 \mathrm{~mm} \times 3 \mathrm{~mm} \times 3 \mathrm{~mm}$. No participant data sets were eliminated in this step. A regression analysis was also done to minimize distortion due to head motion (Friston 24 models), cerebrospinal fluid, and white matter. No participants had head motion exceeding $1.0 \mathrm{~mm}$ of maximal displacement and 1.0 of maximal rotation in any direction. High-frequency physiological noise and low-frequency drift were subsequently filtered $(0.01-0.08 \mathrm{~Hz})$.

ReHo was calculated based on the assumption that voxels within the functional brain region were synchronized with the BOLD time series. Kendall's coefficient of concordance (KCC) was used to measure ReHo of the time series of a given voxel with those of its nearest 26 neighbors in a voxel-wise way. The degree of regional temporal synchronization of the cluster was modeled using the ReHo value applied to a given voxel in the cluster center. For optimization, each ReHo map was divided by the average KCC of the brain. Then, the standardized ReHo map was spatially smoothed at 8-mm fullwidth using a half-maximum (FWHM) Gaussian kernel.

ReHo calculation: The ReHo algorithm measures voxel-wise short-distance FC with Kendall's coefficient of concordance using the following formula:

$$
W=\frac{\sum_{i=1}^{N} R_{i}^{2}-N \bar{R}^{2}}{\frac{1}{12} K^{2}\left(N^{3}-N\right)}
$$

where W is Kendall's coefficient of concordance among the given voxels, $\mathrm{N}$ denotes the length of the time series, $\mathrm{K}=27$ is the size of the voxel cluster containing $3 \times 3 \times 3$ adjacent voxels, Ri denotes the summation of the rankings of the BOLD signal amplitude of all $\mathrm{K}$ voxels at the ith time point, and $\mathrm{R}$ is the mean of $\mathrm{Ri}$.

\section{Statistical Comparisons and Correlations Analysis}

To analyze the statistical differences between the PD-MCI, PD-CN, and NC groups, we performed analysis of covariance (ANCOVA) tests on the ReHo maps and post-hoc analysis to test pairwise significance. The effect of size was calculated using partial eta-squared ( $\eta 2$ ) multiple comparisons correction according to the Bonferroni method. During ANCOVA analysis, we regressed out the age, gender, education years, scores of HAMA and HAMD.

In addition, we assessed the significance of gender with a chi-square $(\chi 2)$ test and that of age, and educational level using an ANOVA. Additionally, post-hoc pairwise comparison was performed with the Bonferroni method. The respective scores for MMSE, MOCA, HAMA, and HAMD were analyzed by Kruskal-Wallis test. The Mann-Whitney $U$-test was followed to detect differences between two groups. The Mann-Whitney $U$-test was used to compare disease duration, LEDD, scores of Revised H-Y, and UPDRS III between the PD$\mathrm{MCI}$ and PD-CN groups. Spearman rank correlations were performed to determine whether the ReHo metrics were associated with clinical indicators (scores of Revised H-Y, UPDRS III, LEDD, MMSE, MOCA, HAMA, and HAMD), and mean ReHo values from the clusters exhibiting significant group differences were assessed as independent variables with age, gender, and years of education as covariates. The BenjaminiHochberg correction was applied to control the false discovery rate. Variables were assessed for normality with Kolmogorov-Smirnov test. All of the statistical thresholds were set at 0.05 (2-tailed). All statistical comparisons were conducted with SPSS 22 statistical software (SPSS Inc., Chicago, III., USA) and Statistical Parametric Mapping, version12 (SPM12, http://www.fil. ion.ucl.ac.uk/spm) running on MATLAB (MathWorks, Inc., Natick, MA, USA).

\section{Results}

\section{Demographics and Clinical Data}

Demographics and clinical data for each group are provided in Table 1. No significant differences among the three groups were found with respect to age or gender $(P>0.05)$, nor were there significant differences between the PD-MCI and PD-CN groups with respect to disease duration, revised $\mathrm{H}-\mathrm{Y}$ scores and LEDD $(P>0.05)$. Significant differences between the PD-MCI and PD-CN groups were found for the UPDRS III score and years of education $(P<0.05)$. Statistically significant differences between the three groups were observed in the years of education and in the scores of MMSE, MOCA, HAMA, 
Table I Demographic and Clinical Data in PD-MCI, PD-CN and NC Group

\begin{tabular}{|c|c|c|c|c|c|c|}
\hline \multirow[t]{2}{*}{ Demographic and Clinical } & \multirow[t]{2}{*}{ PD-MCI $(n=30)$} & \multirow[t]{2}{*}{ PD-CN (n=19) } & \multirow[t]{2}{*}{$N C(n=2 I)$} & \multicolumn{3}{|c|}{$P$} \\
\hline & & & & PD-MCl vs NC & PD-CN vs NC & PD-MCI vs PD-CN \\
\hline Age (years) $^{\mathrm{a}}$ & $61.37 \pm 11.37$ & $58.74 \pm 10.93$ & $60.76 \pm 5.22$ & 0.65 & 0.32 & 0.57 \\
\hline Gender $(F / M)^{b}$ & $14 / 16$ & $5 / 14$ & $13 / 8$ & 0.08 & 0.06 & 0.08 \\
\hline Education (years) ${ }^{a}$ & $8.00 \pm 4.06$ & $11.11 \pm 3.02$ & $9.76 \pm 4.09$ & 0.10 & 0.09 & 0.02 \\
\hline Disease duration (years) ${ }^{c}$ & $3.1(1.5-6.5)$ & $2.5(1.0-4.7)$ & NA & NA & NA & 0.4 \\
\hline Revised H-Y score ${ }^{c}$ & $2.4(1.0-3.0)$ & $1.5(1.0-2.5)$ & NA & NA & NA & 0.06 \\
\hline UPDRS III ${ }^{c}$ & $30.55(17.11-40.22)$ & $21.34(\mid 5.24-29.56)$ & NA & NA & NA & 0.01 \\
\hline LEDD $^{c}$ & $181.58(|1| .02-434)$ & $168.01(50.03-300.00)$ & NA & NA & NA & 0.83 \\
\hline HAMA $^{d}$ & $9(7-17)$ & $10(6-17)$ & $3(2-5)$ & 0.00 & 0.00 & 0.98 \\
\hline HAMD $^{d}$ & $10(6-15)$ & $8(5-16)$ & $4(2-6)$ & 0.00 & 0.00 & 0.90 \\
\hline MMSE $^{d}$ & $26(24-28)$ & $28(27-29)$ & $29(27-30)$ & 0.00 & 0.75 & 0.00 \\
\hline $\mathrm{MoCA}^{\mathrm{d}}$ & $22(19-25)$ & $27(26-29)$ & $27(26-29)$ & 0.00 & 0.89 & 0.00 \\
\hline
\end{tabular}

Notes: Parametric data are shown as means \pm standard deviations. Non-parametric data are shown as median (interquartile range). ${ }^{a} P$ values were calculated using one-way analysis of variance; ${ }^{b} P$ values were calculated using chi-square $\left(\chi^{2}\right)$ test, post-hoc pairwise comparison: the Bonferroni method; ${ }^{c} P$ values were calculated using MannWhitney $U$-test; ${ }^{d} P$ values were calculated using Kruskal-Wallis test.

Abbreviations: PD, Parkinson's disease; NC, normal controls; PD-MCl, PD patients with mild cognitive impairment; PD-CN, PD patients with cognitively normal; NA, nonavailable; F, female; M, male; Revised H-Y score, Revised Hoehn and Yahr score; UPDRS III, Unified Parkinson's Disease Rating Scale motor subscore; LEDD, levodopa equivalent daily dose; HAMA, Hamilton Anxiety Rating Scale; HAMD, Hamilton Depression Rating Scale; MoCA, Montreal Cognitive Assessment; MMSE, Mini-Mental State Examination.

and HAMD $(P<0.05)$. No differences were detected in the MMSE and MoCA scores between of the PD-CN and NC groups $(P>0.05)$. Significant differences in the MMSE and MoCA scores were observed between the PD-MCI and PD-CN groups $(P<0.05)$ and between the PD-MCI and NC groups $(P<0.05)$. Significant differences were also observed in the HAMA and HAMD scores between the PD-MCI and NC groups $(P<0.05)$ and between the PD-CN and NC groups $(P<0.05)$.

\section{ReHo Analyses}

Compared to the NC group, the PD-CN group had significantly higher ReHo values in the right middle frontal gyrus (MFG) and significantly lower ReHo values in the left supramarginal gyrus (SMG), bilateral inferior parietal lobule (IPL), and the right postcentral gyrus (PCG) (Figure 1 and Table 2).

Compared to the PD-CN group, the PD-MCI group exhibited significantly higher ReHo values in the left middle occipital gyrus (MOG), the left IPL, and the right PCG (Figure 2 and Table 2).

\section{Correlation Between the ReHo Values and Clinical Data}

No significant correlations between the ReHo values and the clinical data (disease duration, scores of Revised H-Y, LEDD, MMSE, MOCA, HAMA, and HAMD) were found in the three groups.

\section{Discussion}

In the present work, we attempted to investigate how changes in local neural synchronization differed between NC, PD-CN, and PD-MCI groups using the ReHo method.

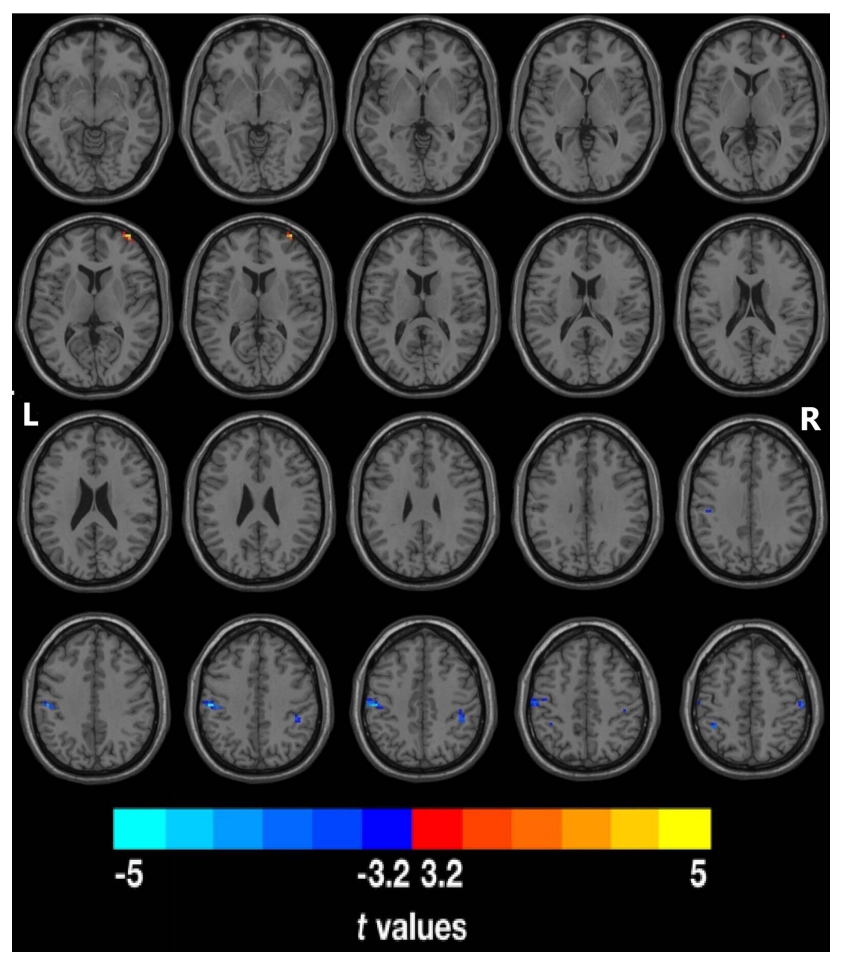

Figure I Changes in ReHo in the PD-CN group compared with the NC group $(P<0.05$, corrected with Bonferroni method). Regions with significantly increased ReHo included the right middle frontal gyrus (MFG). Regions with significantly decreased ReHo included the left supramarginal gyrus (SMG), bilateral inferior parietal lobule (IPL), and right postcentral gyrus (PCG). 
Table 2 Regions Showing ReHo Differences Among the PD-MCl, PD-CN and NC Groups $(P<0.05$, Corrected with Bonferroni Method)

\begin{tabular}{|c|c|c|c|c|c|}
\hline \multirow[t]{2}{*}{ Brain Region } & \multirow[t]{2}{*}{ Voxel Size } & \multicolumn{3}{|c|}{ MNI Coordinaties } & \multirow[t]{2}{*}{ Peak $t$ value } \\
\hline & & $\mathbf{x}$ & $\mathbf{Y}$ & $\mathbf{Z}$ & \\
\hline \multicolumn{6}{|l|}{ PD-CN vs NC } \\
\hline $\mathrm{R}$ middle frontal gyrus & 12 & 36 & 63 & 9 & 3.48 \\
\hline L supramarginal gyrus & 44 & -54 & -21 & 42 & $-4.6 \mid 4$ \\
\hline $\mathrm{R}$ inferior parietal lobule & 14 & 45 & -36 & 45 & -3.499 \\
\hline$L$ inferior parietal lobule & 13 & -42 & -45 & 54 & -4.239 \\
\hline R postcentral gyrus & 24 & 54 & -21 & 54 & -4.03 \\
\hline \multicolumn{6}{|l|}{ PD-MCI vs PD-CN } \\
\hline L middle occipital gyrus & 13 & -33 & $-8 I$ & 18 & 3.930 \\
\hline L inferior parietal lobule & 18 & -39 & -45 & 51 & 4.210 \\
\hline R postcentral gyrus & 10 & 57 & -21 & 51 & 3.96 \\
\hline \multicolumn{6}{|l|}{$\mathrm{PD}-\mathrm{MCl}$ vs $\mathrm{NC}$} \\
\hline None & & & & & \\
\hline
\end{tabular}

Abbreviations: L, left; R, right; MNI, Montreal Neurological Institute.

Our findings revealed that the PD-CN group underwent significant decreases in ReHo values in the left SMG and bilateral IPL within the DMN, but increases in ReHo values in the right $\mathrm{MFG}$ in comparison with NC. Our data also showed that ReHo values in the PD-MCI group

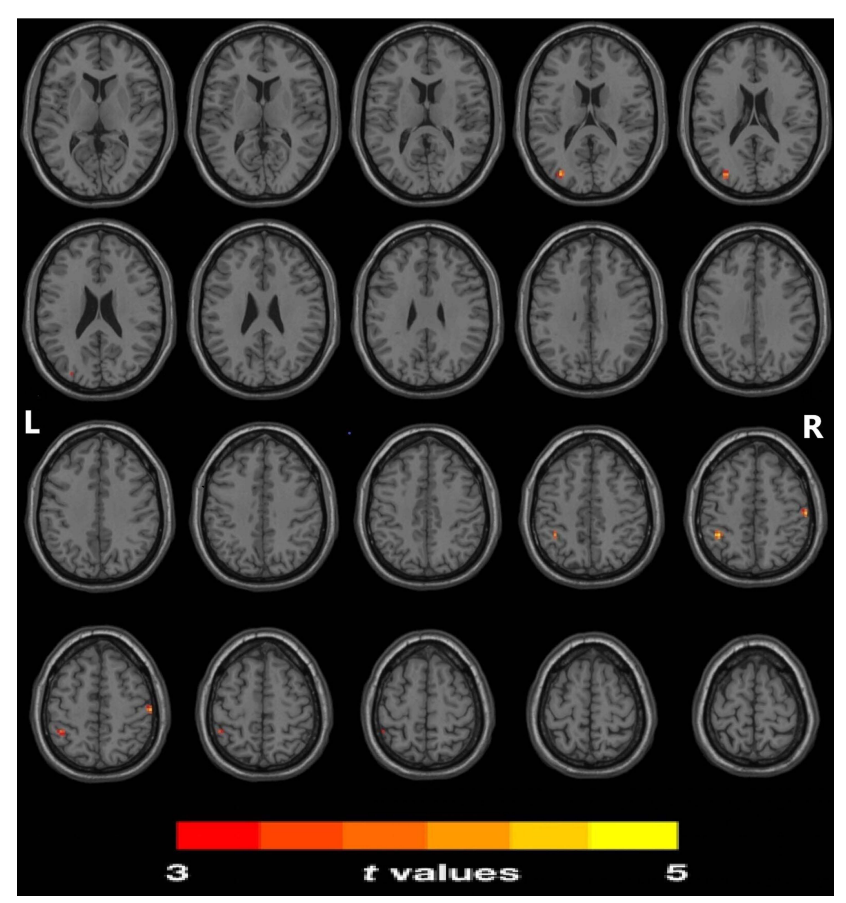

Figure 2 Changes in ReHo in the PD-MCl group compared with the PD-CN group $(P<0.05$, corrected with Bonferroni method). Regions of significantly increased ReHo included the left middle occipital gyrus (MOG), the left inferior parietal lobule (IPL), and the right postcentral gyrus (PCG). were significantly increased in the left $\mathrm{MOG}$ and IPL when compared with that in the PD-CN group.

The PD-CN group showed significantly lower ReHo values in the left SMG and bilateral IPL when compared with NC. Within the DMN, SMG and IPL are both main components. The DMN is thought to be involved in mindwandering, monitoring the world around us, and integration of cognitive and emotional processing. In addition to effects seen in normal aging, the DMN also appears to be involved in the etiopathology of neurodegenerative diseases such as PD, Alzheimer's disease, and Huntington's disease. ${ }^{10,21,22}$ In agreement with previous studies, our study showed decreased ReHo in the DMN in the PD$\mathrm{CN}$ group. For example, using ICA analysis, studies on PD-CN patients have shown decreasing FC of the IPL within the DMN that was positively correlated with visualspatial performance. ${ }^{22,23}$ Using degree centrality (DC), freezing of gait (FOG) of PD-CN patients has also been correlated with DMN changes indicating the presence of pathological damage. ${ }^{24}$ Evidence from ASL research on cognitively normal patients with PD showed that hypoperfusion in the left SMG was negatively correlated with global cognitive performance, consistent with our result. ${ }^{3}$ ReHo presumes that the hemodynamic characteristics of each voxel are synchronized with that of every other within a functional cluster, and this synchronization can be altered or adjusted by different conditions. Decreased ReHo values indicate that local neural synchronization deviate from the NC. It is surmised that hypoactivation 
of the DMN may be due to poor regulation of local neural synchronization indicating PD-related damage. ${ }^{9,24}$ Thus, we posit that the decreased ReHo in DMN may be related to the cognitive impairment of PD and may play a role in the development of PD-associated cognitive decline.

$\mathrm{ReHo}$ in the right MFG was significantly increased in the PD-CN group. The MFG is a major component of the dorsolateral prefrontal cortex, which mediates higher-order cognitive functions such as executive attention, motor planning, decision making, and theory of mind. ${ }^{25,26}$ Our results were consistent with previous research showing that PD patients increased the fractional amplitude of lowfrequency fluctuations (fALFF) or ReHo values in the right MFG compared with $\mathrm{NC}$, which reflected a compensatory mechanism for cognitive dysfunction enabling the retention of cognitive performance. ${ }^{9,27}$ The higher intrinsic $\mathrm{FC}$ of the right MFG has also been reported in dyslexia. ${ }^{26}$ PD-CN patients with FOG showed a higher DC value in the right MFG than the NC indicating that PD-FOG has significant cognitive dysfunction involving frontal lobe executive impairment. ${ }^{24}$ The right MFG is reported to be involved in decision-making in PD. ${ }^{28}$ Increased ReHo in the right MFG may, therefore, compensate for dysfunctional decision-making and loss of executive function, so the PD-CN patients may lack any visible signs on cognitive decline.

In our study, the ReHo of the DMN subsystems decreased but the right MFG increased when comparing PD-CN patients with NC. In the normal subjects, some scholars reported the DMN deactivated and right frontal and parietal cortex activated during attentional lapses. ${ }^{29}$ Increased ReHo of the right MFG in PD patients might reflect compensation for impaired executive function., Interruption of the dynamic equilibrium between the MFG and the DMN may reduce the ability of the cognitive system to prepare for future task execution in PD-FOG patients. $^{24}$ Just like Peter, Boord and colleagues saw reduced resting connectivity to the DMN in the PDnondemented patients but more activation to the frontoparietal network during increased executive challenge. ${ }^{31}$ Taken together, the hyperactivation of the right MFG and hypoactivation of DMN may be a compensatory mechanism for counteracting cognitive decline prior to the appearance of MCI in PD due to executive deficits.

PD-MCI patients showed significantly higher ReHo in the left MOG when compared with PD-CN patients. PD patients exhibited a variety of problems in vision or visualspatial function such as visual hallucination or even early stages of FOG. ${ }^{32}$ The occipital lobe is essential to memory-related, visual-spatial information processing and in perceptive priming, ${ }^{33,34}$ and MOG is a component of the visual cortex. PD patients with visual hallucination display abnormal ALFF values in the visual cortex. ${ }^{35}$ Additionally, increased ReHo values are observed in the left visual cortex of PD patients with PIGD in comparison with TD, which may be associated with visual deficits. ${ }^{16}$ Finally, both PD patients off medication and PD-MCI patients depicted increased ReHo in the left MOG in comparison with NC. ${ }^{13,15,16}$ In other words, it is possible that the ReHo alterations observed in the left MOG may be related to a dysfunction of vision and visual-spatial capacity in PDMCI patients.

PD-MCI patients showed significantly higher ReHo in the left IPL when compared with PD-CN patients. It is known that the IPL takes part in various cognitive functions such as spatial attention, language processing, and episodic memory retrieval. ${ }^{36,37}$ The IPL is also a sensory motor interface for integration of sensory feedback, especially visual and proprioceptive information. ${ }^{38}$ Our observations align with those of prior studies on MCI, multiple sclerosis and PD patients. Evidence from research on MCI showed increases in ReHo values and intrinsic connectivity in the IPL, findings which may reflect overwork in network resources and compensatory recruitment for early-stage cognitive deficits. ${ }^{10,39}$ In the patients with multiple sclerosis, the connectivity of inferior parietal gyrus increased with worse cognition which maybe caused by cortical plasticity or reduction in white matter integrity. ${ }^{40}$ PD patients having FOG showed increased ALFF in the left IPL compared to those without FOG. ${ }^{41}$ Increased ReHo values were observed in the left IPL in PD patients even in early stages or during visual hallucinations. ${ }^{9,16,35,42}$ Using ICA analysis, Baggio and colleagues discovered increased connectivity between the DMN and medial and lateral occipito-parietal regions in PD-MCI patients. The disruption of these dynamic coupling mechanisms may worsen visuo-spatial/visuoperceptual performance. ${ }^{4}$ Dopaminergic and cholinergic denervation are the main neurophysiological mechanisms associated with cognitive dysfunction in PD. ${ }^{43}$ RS-fMRI meta-analysis of PD subjects revealed abnormal intrinsic functional patterns located mainly within the posterior part of the left IPL that constitutes a compensatory process for countering inefficient processing within the basal ganglia and that is due to dopamine depletion. ${ }^{44}$ Together, we conclude that the increased ReHo in the left IPL which 
contributes to cognitive dysfunction, may be due to the neuropathological damage.

The PD-CN group showed significantly lower ReHo values in the right PCG when compared with NC. The PCG is a cortical core in somatosensory processing, involving, for example, the somatic sensoria for external stimuli. $^{45}$ It receives sensory input subsequent to motorexecution for successive fine-tuning. Deficits in the perception of painful, tactile, thermal, and proprioceptive inputs, moreover, are common in $\mathrm{PD} .{ }^{46}$ Also, repetitive transcranial stimulation can access the PCG as a stimulation target to alleviate clinical symptoms. ${ }^{47}$ The neuronal dysfunction of the sensorimotor cortex in striatalthalamo-cortical loops can predominate in the initial stages of PD and cause motor difficulties. ${ }^{48}$ Accordingly, the decreased ReHo in the right PCG may be related to an early stage of PD. ${ }^{13}$ Hypoactivation in the right PCG of PD patients has been observed in previous RS-fMRI studies, ${ }^{13,17,24}$ whereas the PD-MCI group showed significantly higher ReHo values in the right PCG when compared with the PD-CN group. Overactivation of the sensorimotor cortex has been reported in PD and MCI patients. Some scholars posit that the increased ReHo in the PCG may be interpreted as a compensation for cognitive function decline, ${ }^{8,16}$ while others propose that the cortical reorganization after anti-parkinsonian medication in the later stage of PD plays a part in the increased ReHo in the PCG. ${ }^{49}$ In sum, the decrease in ReHo in the right PCG of the PD-CN group may be responsible for the disruption of somatosensory processing, and the increase in ReHo in the PD-MCI group may be responsible for a compensation of cognitive dysfunction or cortical restoration after dopamine replacement.

Our study has several limitations. First, the sample size of each group is relatively small. Small sample size can limit identification of minor local neural synchronization that correlates with MCI in PD and increases the rate of false-negatives. ${ }^{8,24}$ In the future, a larger sample size of PD patients will be recruited to strengthen our results. Second, there are both drug-naïve and regular dopaminergic replacement therapy PD patients for conducting this research. The potentially confounding effects of dopaminergic treatment on cognitive function are still being debated. ${ }^{50}$ Nevertheless, our correlation analysis did not show any significant association between ReHo indices and the LEDD. Third, in our research we adopted limited cognitive evaluation. Evaluation of different cognitive domains usually requires longitudinal studies, which are difficult for patients to undergo. In our study, we chose two global cognitive scales that were nonspecific. In the future, if our subjects can participate, comprehensive neuropsychological examinations should be adopted. Fourth, the PD-CN group had more years of education (11.11 \pm 3.02$)$ compared with that of PD-MCI $(8.00 \pm 4.06)$ and $\mathrm{NC}(9.76 \pm 4.09)$ groups. Higher education levels were generally associated with significantly better cognitive performance and a small but significant slowing in cognitive decline. ${ }^{51}$ We have already taken these factors into account in our statistical analysis. Therefore, we can limit the impact of the education-year in our conclusions from the two subgroups as far as possible. Finally, the present research is cross-sectional. In the long run, longitudinal investigations may provide insight into the causal relationship between the progression of cognitive impairment and ReHo changes. This may be due to the above factors, in which no significant differences were found between PD-MCI patients and NC. Unexpectedly, there was no significant correlation between the ReHo values and the neuropsychological scales also.

\section{Conclusion}

In summary, our research on ReHo values obtained from the DMN demonstrated an impairment before cognitive deterioration occurred in PD subjects. The increased ReHo in the right MFG may be attributed to maintain executive ability in PD-CN patients. The present study revealed that the increased ReHo in the left MOG may be related to a dysfunction of vision and visual-spatial ability. The increased ReHo in the left IPL may be caused by neuropathological changes and resulted in cognitive deficits in PD-MCI patients. The ReHo changes in spontaneous regional brain activity may be useful for characterizing the neurophysiology of PD-MCI and may be a valuable method for the early detection of PD-MCI.

\section{Acknowledgments}

The authors thank all the participants and staff involved in this study. This work was supported by the National Natural Science Foundation of China (Grant Nos. 81771807 and 81901729) and the Science and Technology Planning Project of Guangzhou (Grant Nos. 202002030234). 


\section{Author Contributions}

All authors made a significant contribution to the work reported, whether that is in the conception, study design, execution, acquisition of data, or analysis and interpretation, or in all these areas; took part in drafting, revising or critically reviewing the article; gave final approval of the version to be published; have agreed on the journal to which the article has been submitted; and agree to be accountable for all aspects of the work.

\section{Disclosure}

Dr Yilan Xing reports grants from the National Natural Science Foundation of China, grants from the Science and Technology Planning Project of Guangzhou, during the conduct of the study. The authors declare no conflicts of interest.

\section{References}

1. Jones JD, Kuhn TP, Szymkowicz SM. Reverters from PD-MCI to cognitively intact are at risk for future cognitive impairment: analysis of the PPMI cohort. Parkinsonism Relat Disord. 2018;47:3-7. doi:10.1016/j.parkreldis.2017.12.006

2. Wang Q, Zhang Z, Li L, Wen H, Xu Q. Assessment of cognitive impairment in patients with Parkinson's disease: prevalence and risk factors. Clin Interv Aging. 2014;9:275-281.

3. Syrimi ZJ, Vojtisek L, Eliasova I, et al. Arterial spin labelling detects posterior cortical hypoperfusion in non-demented patients with Parkinson's disease. J Neural Transm. 2017;124(5):551-557. doi:10.1007/s00702-017-1703-1

4. Baggio H, Segura B, Sala-Llonch R, et al. Cognitive impairment and resting-state network connectivity in Parkinson's disease. Hum Brain Mapp. 2015;36(1):199-212. doi:10.1002/hbm.22622

5. Hou Y, Yang J, Luo C, et al. Dysfunction of the default mode network in drug-naive parkinson's disease with mild cognitive impairments: a resting-state fMRI study. Front Aging Neurosci. 2016;8:247. doi:10.3389/fnagi.2016.00247

6. Li Z, Kadivar A, Pluta J, Dunlop J, Wang Z. Test-retest stability analysis of resting brain activity revealed by blood oxygen level-dependent functional MRI. J Magn Reson Imaging. 2012;36 (2):344-354. doi:10.1002/jmri.23670

7. Zuo $\mathrm{X}, \mathrm{Xu} \mathrm{T}$, Jiang $\mathrm{L}$, et al. Toward reliable characterization of functional homogeneity in the human brain: preprocessing, scan duration, imaging resolution and computational space. Neuroimage. 2013;65:374-386. doi:10.1016/j.neuroimage.2012.10.017

8. Li J, Yuan Y, Wang M, et al. Alterations in regional homogeneity of resting-state brain activity in fatigue of Parkinson's disease. J Neural Transm. 2017;124(10):1187-1195. doi:10.1007/s00702-017-1748-1

9. Pan P, Zhan H, Xia M, Zhang Y, Guan D, Xu Y. Aberrant regional homogeneity in Parkinson's disease: a voxel-wise meta-analysis of resting-state functional magnetic resonance imaging studies. Neurosci Biobehav Rev. 2017;72:223-231. doi:10.1016/j.neubiorev.20 16.11 .018

10. Zhang Z, Liu Y, Jiang T, et al. Altered spontaneous activity in Alzheimer's disease and mild cognitive impairment revealed by regional homogeneity. Neuroimage. 2012;59(2):1429-1440. doi:10.1016/j.neuroimage.2011.08.049

11. Guo W, Jin W, Li N, et al. Brain activity alterations in patients with Parkinson's disease with cognitive impairment based on resting-state functional MRI. Neurosci Lett. 2021;747:135672. doi:10.1016/j. neulet.2021.135672
12. Harrington DL, Shen Q, Castillo GN, et al. Aberrant intrinsic activity and connectivity in cognitively normal Parkinson's disease. Front Aging Neurosci. 2017;9(197):197. doi:10.3389/fnagi.2017.00197

13. Choe IH, Yeo S, Chung KC, Kim SH, Lim S. Decreased and increased cerebral regional homogeneity in early Parkinson's disease. Brain Res. 2013;1527:230-237.

14. Li M, Liu T, Zhang T, et al. Alterations of regional homogeneity in Parkinson's disease with mild cognitive impairment: a preliminary resting-state fMRI study. Neuroradiology. 2020;62(3):327-334. doi:10.1007/s00234-019-02333-7

15. Li Y, Liang $\mathrm{P}$, Jia $\mathrm{X}$, Li K. Abnormal regional homogeneity in Parkinson's disease: a resting state fMRI study. Clin Radiol. 2016;71(1):e28-e34

16. Jiang S, Wang M, Zhang L, et al. Regional homogeneity alterations differentiate between tremor dominant and postural instability gait difficulty subtypes of Parkinson's disease. J Neural Transm. 2016;123(3):219-229.

17. Zeng Q, Guan X, Law YLJ, et al. Longitudinal alterations of local spontaneous brain activity in Parkinson's disease. Neurosci Bull. 2017;33(5):501-509. doi:10.1007/s12264-017-0171-9

18. Litvan I, Goldman JG, Troster AI, et al. Diagnostic criteria for mild cognitive impairment in Parkinson's disease: movement disorder society task force guidelines. Mov Disord. 2012;27(3):349-356. doi:10.1002/mds. 24893

19. Hughes AJ, Daniel SE, Kilford L, Lees AJ. Accuracy of clinical diagnosis of idiopathic Parkinson's disease: a clinico-pathological study of 100 cases. J Neurol Neurosurg Psychiatry. 1992;55 (3):181-184. doi:10.1136/jnnp.55.3.181

20. Kim JI, Sunwoo MK, Sohn YH, Lee PH, Hong JY. The MMSE and MoCA for screening cognitive impairment in less educated patients with Parkinson's disease. J Mov Disord. 2016;9(3):152-159. doi:10.14802/jmd.16020

21. Wolf RC, Sambataro F, Vasic N, et al. Default-mode network changes in preclinical Huntington's disease. Exp Neurol. 2012;237 (1):191-198. doi:10.1016/j.expneurol.2012.06.014

22. Hou Y, Yang J, Luo C, et al. Resting-state network connectivity in cognitively unimpaired drug-naive patients with rigidity-dominant Parkinson's disease. J Neurol Sci. 2018;395:147-152. doi:10.1016/j. jns.2018.10.003

23. Tessitore A, Esposito F, Vitale C, et al. Default-mode network connectivity in cognitively unimpaired patients with Parkinson disease. Neurology. 2012;79(23):2226-2232. doi:10.1212/WNL.0b013e3 $1827689 \mathrm{~d} 6$

24. Guo M, Ren Y, Yu H, et al. Alterations in degree centrality and functional connectivity in Parkinson's disease patients with freezing of gait: a resting-state functional magnetic resonance imaging study. Front Neurosci. 2020;14:582079. doi:10.3389/fnins.2020.582079

25. Lau H, Shahar S, Mohamad M, et al. Relationships between dietary nutrients intake and lipid levels with functional MRI dorsolateral prefrontal cortex activation. Clin Interv Aging. 2019;14:43-51. doi:10.2147/CIA.S183425

26. Mateu-Estivill R, Forne S, Lopez-Sala A, et al. Functional connectivity alterations associated with literacy difficulties in early readers. Brain Imaging Behav. 2020:1-2.doi:10.1007/s11682-020-00406-32

27. Hu XF, Zhang JQ, Jiang XM, et al. Amplitude of low-frequency oscillations in Parkinson's disease: a 2-year longitudinal restingstate functional magnetic resonance imaging study. Chin Med J. 2015;128(5):593-601. doi:10.4103/0366-6999.151652

28. Kobayakawa M, Tsuruya N, Kawamura M. Decision-making performance in Parkinson's disease correlates with lateral orbitofrontal volume. $J$ Neurol Sci. 2017;372:232-238. doi:10.1016/j. jns.2016.11.046

29. Weissman DH, Roberts KC, Visscher KM, Woldorff MG. The neural bases of momentary lapses in attention. Nat Neurosci. 2006;9 (7):971-978. doi:10.1038/nn1727 
30. Wu T, Hallett M. A functional MRI study of automatic movements in patients with Parkinson's disease. Brain. 2005;128(Pt10):2250-2259. doi:10.1093/brain/awh569

31. Boord P, Madhyastha TM, Askren MK, Grabowski TJ. Executive attention networks show altered relationship with default mode network in PD. Neuroimage Clin. 2017;13:1-8. doi:10.1016/j. nicl.2016.11.004

32. Armstrong RA. Visual dysfunction in Parkinson's disease. Int Rev Neurobiol. 2017;134:921-946.

33. He X, Zhang Y, Chen J, et al. Changes in theta activities in the left posterior temporal region, left occipital region and right frontal region related to mild cognitive impairment in Parkinson's disease patients. Int $J$ Neurosci. 2017;127(1):66-72. doi:10.3109/ 00207454.2016 .1143823

34. De Marco M, Duzzi D, Meneghello F, Venneri A. Cognitive efficiency in Alzheimer's disease is associated with increased occipital connectivity. J Alzheimers Dis. 2017;57(2):541-556. doi:10.3233/ JAD-161164

35. Yao N, Pang S, Cheung C, et al. Resting activity in visual and corticostriatal pathways in Parkinson's disease with hallucinations. Parkinsonism Relat Disord. 2015;21(2):131-137. doi:10.1016/j. parkreldis.2014.11.020

36. Sestieri C, Corbetta M, Romani GL, Shulman GL. Episodic memory retrieval, parietal cortex, and the default mode network: functional and topographic analyses. $J$ Neurosci. 2011;31(12):4407-4420. doi:10.1523/JNEUROSCI.3335-10.2011

37. Caspers S, Schleicher A, Bacha-Trams M, Palomero-Gallagher N, Amunts K, Zilles K. Organization of the human inferior parietal lobule based on receptor architectonics. Cereb Cortex. 2013;23 (3):615-628. doi:10.1093/cercor/bhs048

38. Sober SJ, Sabes PN. Flexible strategies for sensory integration during motor planning. Nat Neurosci. 2005;8(4):490-497. doi:10.1038/ nn1427

39. Esposito R, Mosca A, Pieramico V, Cieri F, Cera N, Sensi SL. Characterization of resting state activity in MCI individuals. Peerj. 2013;1:e135.

40. Meijer KA, Eijlers A, Douw L, et al. Increased connectivity of hub networks and cognitive impairment in multiple sclerosis. Neurology. 2017;88(22):2107-2114. doi:10.1212/WNL.0000000000003982
41. Mi TM, Mei SS, Liang PP, et al. Altered resting-state brain activity in Parkinson's disease patients with freezing of gait. Sci Rep. 2017;7 (1):16711. doi:10.1038/s41598-017-16922-0

42. Yang H, Zhou XJ, Zhang MM, Zheng XN, Zhao YL, Wang J. Changes in spontaneous brain activity in early Parkinson's disease. Neurosci Lett. 2013;549:24-28. doi:10.1016/j.neulet.2013.05.080

43. Kalia LV. Biomarkers for cognitive dysfunction in Parkinson's disease. Parkinsonism Relat Disord. 2018;46(Suppl 1):S19-S23. doi:10.1016/j.parkreldis.2017.07.023

44. Tahmasian M, Eickhoff SB, Giehl K, et al. Resting-state functional reorganization in Parkinson's disease: an activation likelihood estimation meta-analysis. Cortex. 2017;92:119-138. doi:10.1016/j. cortex.2017.03.016

45. Nelson AJ, Chen R. Digit somatotopy within cortical areas of the postcentral gyrus in humans. Cereb Cortex. 2008;18(10):2341-2351. doi:10.1093/cercor/bhm257

46. Conte A, Khan N, Defazio G, Rothwell JC, Berardelli A. Pathophysiology of somatosensory abnormalities in Parkinson disease. Nat Rev Neurol. 2013;9(12):687-697. doi:10.1038/ nrneurol.2013.224

47. Ji GJ, Hu P, Liu TT, et al. Functional connectivity of the corticobasal ganglia-thalamocortical network in Parkinson disease: a systematic review and meta-analysis with cross-validation. Radiology. 2018;287 (3):973-982. doi:10.1148/radiol.2018172183

48. Sharman M, Valabregue R, Perlbarg V, et al. Parkinson's disease patients show reduced cortical-subcortical sensorimotor connectivity. Mov Disord. 2013;28(4):447-454. doi:10.1002/mds.25255

49. Wu T, Long X, Zang Y, et al. Regional homogeneity changes in patients with Parkinson's disease. Hum Brain Mapp. 2009;30 (5):1502-1510. doi:10.1002/hbm.20622

50. Zhong J, Guan X, Zhong X, et al. Levodopa imparts a normalizing effect on default-mode network connectivity in non-demented Parkinson's disease. Neurosci Lett. 2019;705:159-166. doi:10.1016/ j.neulet.2019.04.042

51. Hindle JV, Martyr A, Clare L. Cognitive reserve in Parkinson's disease: a systematic review and meta-analysis. Parkinsonism Relat Disord. 2014;20(1):1-7. doi:10.1016/j.parkreldis.2013.08.010
Neuropsychiatric Disease and Treatment

\section{Publish your work in this journal}

Neuropsychiatric Disease and Treatment is an international, peerreviewed journal of clinical therapeutics and pharmacology focusing on concise rapid reporting of clinical or pre-clinical studies on a range of neuropsychiatric and neurological disorders. This journal is indexed on PubMed Central, the 'PsycINFO' database and CAS, and is the official journal of The International Neuropsychiatric Association (INA). The manuscript management system is completely online and includes a very quick and fair peer-review system, which is all easy to use. Visit http://www.dovepress.com/testimonials.php to read real quotes from published authors. 